\title{
PRIMERA INFANCIA EN RIESGO: LAS PRÁCTICAS DE CRIANZA EN BARRANCA HONDA, PUEBLA, MÉXICO
}

\author{
EARLY CHILDHOOD AT RISK: EDUCATION PRACTICES IN BARRANCA HONDA, \\ PUEBLA, MEXICO
}

\section{PRIMEIRA INFÂNCIA EM RISCO: AS PRÁTICAS DE EDUCAÇÃO EM BARRANCA HONDA, PUEBLA, MÉXICO}

\author{
Karla Villaseñor Palma* \\ Claudia Guzmán Zárate*
}

\begin{abstract}
Resumen: Con el objetivo de conocer las prácticas de crianza de los padres y cuidadores de los niños menores de 5 años que viven en la colonia Barranca Honda, comunidad caracterizada por la pobreza y la exclusión social y educativa, se realizó una investigación que permitiera detectar las necesidades de dichos actores para dar pie a estrategias socioeducativas que favorezcan el desarrollo integral de la primera infancia en dicha comunidad. La investigación surge en el marco del trabajo realizado con la Escuela Comunitaria del Centro Universitario de Participación Social de la Benemérita Universidad Autónoma de Puebla, México. El diseño metodológico fue de corte cuasi-etnográfico. Los resultados muestran que las prácticas de crianza de padres y cuidadores se centran mayoritariamente en atender al desarrollo físico del niño y que las características del contexto impiden la construcción de redes sociales, lo que afecta significativamente las prácticas de crianza de los padres y, por tanto, el desarrollo integral de los niños de esta colonia. Estos hallazgos nos permiten ubicar algunos espacios desde los cuales es posible responder al desafío de romper con la situación de riesgo latente en la que viven y crecen los niños de dicha colonia.
\end{abstract}

Palabras claves:Primera Infancia. Prácticas de crianza. Riesgo. Vulnerabilidad. Cuasi-etnografía.

\begin{abstract}
In order to understand the practices of parents and caregivers of children younger tan 5 years old living in the neighborhood of Barranca Honda, a community characterized by poverty and social and educational exclusion, an investigation was carried out to detect the needs of such actors for to give rise to socio-educational strategies that favor the integral development of early childhood in the said community. The research is part of the work undertaken with the Community School of the University Center of Social Participation at the Autonomous University of Puebla, Mexico. The methodological design was near-ethnographic nature. The results show that parents' and caregivers' educational practices focus mainly on attending to the physical development of the child and that the characteristics of the context prevents the construction of social networks, which significantly affects parents' education practices and, therefore, the integral development of the children of this neighborhood. These discoveries allow us to find some spaces from which is possible to respond to the challenge of breaking with the latent risk situation in which the children of that colony live and grow.
\end{abstract}

Keywords: Early childhood. Education practices. Risk. Vulnerability. Near-ethnography

\footnotetext{
* Professora Doutora na Benemérita Universidad Autónoma de Puebla - México. E-mail: villasenorkarla@gmail.com

** Professora Doutora na Benemérita Universidad Autónoma de Puebla, México.

E-mail: claudia.guzman@correo.buap.mx
} 
Resumo: Com o objetivo de conhecer as práticas de educação de pais e cuidadores de crianças menores de 5 anos que vivem no bairro da Barranca Honda, uma comunidade caracterizada pela pobreza e exclusão social e educacional, foi realizada uma investigação para detectar as necessidades de ditos atores para dar origem a estratégias socioeducativas que favorecem o desenvolvimento integral da primeira infância na dita comunidade. A pesquisa surge no âmbito do trabalho realizado com a Escola Comunitária do Centro Universitário de Participação Social da Universidade Autônoma Benemérita de Puebla, México. O desenho metodológico foi de natureza quase-etnográfica. Os resultados mostram que práticas de educação de pais e cuidadores focam principalmente no atendimento ao desenvolvimento físico da criança e que as características do contexto impedem a construção de redes sociais, o que afeta significativamente as práticas de educação dos pais e, portanto, o desenvolvimento integral dos filhos deste bairro. Essas descobertas nos permitem localizar alguns espaços a partir dos quais é possível responder ao desafio de romper com a situação de risco latente em que os filhos daquela colônia vivem e crescem.

Palavras-chave: Primeira infância. Práticas de educação. Risco. Vulnerabilidade. Quase-etnografia.

\section{Introducción}

En las últimas décadas, la primera infancia ha ocupado un lugar primordial en materia de desarrollo social. Ello se debe, en gran medida, a la vasta producción científica que ha demostrado el valor que para el desarrollo social y de la persona tienen los primeros años de vida. (HECKMAN, 2006; NATIONAL SCIENTIFIC COUNCIL ON THE DEVELOPING CHILD, 2017; UNESCO, 2007; WOODHEAD, 2006; entre otros). Esas evidencias han llevado al estudio de los beneficios personales y sociales que trae consigo el buen desarrollo desde una temprana edad, llegando incluso a considerar que la inversión en el bienestar de la primera infancia es la estrategia de inversión pública más redituable en la actualidad. (HECKMAN, 2006). No obstante, la realidad social en que vivimos priva de esta posibilidad a muchos niños que viven en entornos vulnerables: caracterizados por condiciones de pobreza, falta de acceso a servicios básicos y exclusión social y educativa. Las consecuencias inmediatas de dicha exclusión son bien conocidas: enfermedades, malnutrición, atrofia del crecimiento y disminución del desarrollo motor, lingüístico, cognitivo, social y emocional, entre otras
(SIRAJ-BLATCHFORD; WOODHEAD, 2009); las cuales se traducen en un rezago educativo que genera un ciclo de desigualdad permanente del que difícilmente se puede salir.

En este sentido, uno de los desafíos más importantes para quienes desarrollan políticas y programas dirigidos a la primera infancia y sus familias es lograr los mejores resultados intentado conciliar la evidencia científica sobre lo que un niño necesita, con las prácticas y creencias tradicionales y efectivas de crianza. (EVANS; MYERS, 1994). Un reto que se debe intentar resolver, en la medida de lo posible, de manera previa al diseño de dichas políticas y programas.

\section{Antecedentes: infancia en pobreza y desigualdad en Puebla}

Puebla ocupa el $3^{\circ}$ lugar percentil de población en pobreza y el $4^{\circ}$ en población en pobreza extrema. (CONEVAL, 2013). Ocho de sus municipios se encuentran entre los 100 más pobres del país y aproximadamente el $60 \%$ de la población vive por debajo del umbral de la pobreza. (INEGI, 2010). En Puebla se encuentra uno de los sectores industriales más importantes del país, lo 
cual lo coloca como uno de los estados con mayor desigualdad social en la República (Coeficiente de GINI del 0,5170).

En 2010, el Estado de Puebla tenía 5.779.829 habitantes, de los cuales 574.513 eran menores de 4 años, es decir, el 9\%. En este mismo año el Estado de Puebla registró un crecimiento demográfico del 1,3 y una tasa de natalidad de 18,59. El 18,2\% de los nacimientos ocurridos en este año fueron de madres adolescentes. Además, Puebla tiene una de las tasas de mortalidad infantil más altas del país en menores de uno y cinco años. (INEGI, 2010).

Las características económicas y demográficas del Estado afectan directamente a la primera infancia por lo que el $17,47 \%$ de los niños menores de 5 años que habitan en el Estado padecen pobreza extrema y el 50,1\% pobreza moderada. Según UNICEF México (2010), Puebla junto con Chiapas, Guerrero, Oaxaca y Veracruz, concentra el mayor número de niños y niñas indígenas en condiciones de pobreza alimentaria, cantidad que oscila entre los $100 \mathrm{mil} \mathrm{y} \mathrm{los} 350 \mathrm{mil}$ niños. Y en materia de educación, sólo el $52,6 \%$ de los niños y el 52\% de las niñas de entre 2 y 5 años de edad asiste a la escuela.

\section{El problema: riesgos en Barranca Honda}

"La historia del reparto de los riesgos muestra que estos siguen, al igual que las riquezas, el esquema de clases, pero al revés: las riquezas se acumulan arriba, los riesgos abajo."

(Beck, 1998, p. 40)

El Centro Universitario de Participación Social (CUPS) de la Benemérita Universidad Autónoma de Puebla (BUAP) atiende a niños y adolescentes no escolarizados a través del
Programa “Escuelas Comunitarias”. Una de estas escuelas se ubica en la colonia Barranca Honda, perteneciente a una junta auxiliar del Municipio de Puebla. Dicha colonia fue fundada espontáneamente y sin regulaciones hace apenas unas pocas décadas. No obstante, hoy muestra serios problemas territoriales a los que se suman la pobreza, la marginalidad y la inseguridad en la que viven sus habitantes, en su mayoría menores de edad.

De todas las escuelas comunitarias del CUPS, la Escuela Comunitaria de Barranca Honda es la más concurrida. Asisten a ella más de 40 niños de entre 6 y 14 años que por diversas razones permanecen fuera del sistema educativo.

Considerando que las desigualdades educativas tienen origen en la primera infancia, un periodo de la vida en el que las prácticas de crianza influyen decisivamente (ya sea contrarrestando o favoreciendo dichas desigualdades), interesa conocerlas a fin de comprender mejor las necesidades de cuidadores, padres y niños de la colonia. Para ello se deben conocer las condiciones actuales de los niños de 0 a 5 años que viven este lugar y las prácticas de crianza (acciones, creencias, actitudes) de los adultos de quienes dependen (padres y/o cuidadores).

Según Evans y Myers (1994), es imprescindible conocer y apoyar las prácticas de crianza de padres y cuidadores para mejorar las condiciones de vida de los niños. Este apoyo no puede partir únicamente del conocimiento científico sobre los cuidados prenatales y postnatales, sino que también debe tomar en cuenta la experiencia de las personas, sus valores culturales y sus costumbres. Para estos autores, las prácticas de crianza deben garantizar el bienestar físico del niño; promover su bienestar psicosocial; apoyar el desarrollo físico y mental; y facilitar la interacción del niño con la comunidad. A 
partir de estas consideraciones, se distinguieron dos tipos de prácticas de crianza: a) prácticas de crianza dirigidas a cubrir las necesidades básicas (o de sobrevivencia); y b) prácticas de crianza dirigidas a promover el desarrollo integral del niño. Si bien pareciera que las primeras constituyen el andamiaje básico para el desarrollo del niño, son ciertamente insuficientes. Es más, las prácticas de crianza de sobrevivencia constituyen un riesgo en términos de tiempo.

En este sentido, la noción de riesgo, acuñada por Beck (1998), nos permite hablar en términos de contingencia, es decir, de un evento que todavía no acontece pero que puede suceder. El riesgo implica aceptar que las condiciones están dadas para que algo suceda; y con el fin de comprender mejor la incidencia que las prácticas de crianza de los padres tiene en el desarrollo de los niños y las niñas de la colonia, se analizan a continuación los principales hallazgos de la investigación en este sentido; tanto a partir de las condiciones del contexto que propician dichas prácticas, como a la luz de las propias prácticas que se constituyen en un riesgo para los menores.

\section{Objetivo general de la investigación}

Partimos de la idea, ya demostrada, de que las desigualdades educativas, y en general las desigualdades sociales están presentes desde los primeros años de vida y que incidir en este periodo de la vida puede revertir los efectos del contexto y las condiciones socioeconómicas. Para poder hacerlo mejor, es necesario conocer las condiciones actuales de los niños de 0 a 5 años que viven en Barranca Honda y las prácticas de crianza (acciones, creencias, actitudes) de los adultos de quienes dependen (padres y/o cuidadores). Por lo anterior, nos planteamos el siguiente objetivo de investigación:
Comprender las prácticas, patrones y creencias sobre la crianza de los padres y cuidadores de los niños de 0 a 5 años que viven en la colonia Barranca Honda, así como la relación que guardan con las condiciones de riesgo que presentan dichas familias y que se extienden a los más pequeños.

\section{Metodología}

En correspondencia con los propios objetivos de la investigación, el diseño metodológico del estudio fue cuasietnográfico (SILVA; BURGOS, 2011) cuya premisa principal es que en un tiempo mínimo es posible generar un conocimiento suficiente. Dicha metodología nos permitió conocer y comprender de forma apropiada las prácticas de crianza de padres y cuidadores de niños de 0 a 5 años de Barranca Honda cuyos hijos mayores de 6 años asisten a la escuela comunitaria de la colonia (niños del CUPS). Así mismo, se consideró importante incluir una muestra de padres y cuidadores de niños de 0 a 5 años cuyos hijos mayores de 6 años asisten a la escuela pública de la zona (niños escolarizados) para saber si existían diferencias significativas en dichas prácticas que pudieran explicar las condiciones de escolarización y de no escolarización.

De esta manera, la muestra se determinó a partir de casos extremos conformados por dos tipos de familias:

- Grupo A integrado por seis familias con niños menores de 5 años y hermanos mayores no escolarizados

- Grupo B integrado por seis familias con niños menores de 5 años y hermanos mayores escolarizados.

Dado el tipo de estudio que se realiza, se empleó el muestreo discrecional, -mismo que se utiliza principalmente en las Ciencias 
Sociales y que busca obtener la opinión de expertos, más que una muestra basada en datos estadísticos.

De esta manera, para seleccionar a las familias del primer grupo, se realizó previamente un grupo focal en el que participaron los educadores del CUPS. Si bien el CUPS trabaja con niños de 5 años en adelante, se seleccionaron familias en las que hubiera niños menores y/o mujeres embarazadas. Para seleccionar a las familias del segundo grupo se hicieron entrevistas semiestructuradas a las instructoras de primero y segundo grado de preescolar del Centro de Asistencia Infantil Comunitario (CAIC).

Una vez seleccionadas las familias se realizaron visitas domiciliarias a cada una de las doce familias. Se presentaron en cada hogar un investigador de la Facultad de Filosofía y Letras de la BUAP asistido por un estudiante de la Licenciatura en Procesos Educativos. El investigador realizó la entrevista semiestructurada (apoyándose de un guion de entrevista, un familiograma y una hoja de rutina diaria), al tiempo que el estudiante realizó la observación no participante utilizando el marco AEIOU: Activities-actividades, Environmentsambiente, Interactions-interacciones, Objectsobjetos y Users-usuarios. (ROBINSON; PROKOPOFF; CAIN; POKORNY, 1991 apud HANINGTON; MARTIN, 2012).

\section{Resultados de la investigación: las prácticas de crianza en Barranca Honda}

\section{El contexto: un espacio caracterizado por altos riesgos}

La colonia Barranca Honda pertenece a la junta auxiliar de San Pablo Xochimehuacan del municipio de Puebla. Dicha junta auxiliar perteneció al señorío de Cholula, por lo que su población es antigua y está emparentada con ese importante centro ceremonial y cultural. Entre sus principales actividades destacaban la agricultura y la alfarería; además de corresponder a su pacto tributario con los cholultecas custodiando el territorio de posibles invasiones de otros grupos como los totonacas, los mixtecos, los popolocas y los tlaxcaltecas. Hoy existen en ella familias del grupo náhuatl que también hablan castellano, y predomina el mestizaje. Su territorio fue municipio libre en el año 1930, con cabecera en San Pablo Xochimehuacan; y en el año 1963, por decreto del H. XLI Congreso Constitucional del Estado de Puebla, se anexa al Municipio de Puebla como Junta Auxiliar. (CARRILLO VIVAS, 1996, p. 17). Año en el que el gobierno Federal expropió tierras para construir el Mercado de Abastos. La expropiación de tierras y la veta de terrenos irregulares han marcado fuertemente a la población de esta zona, figurando entre su historia reciente los conflictos políticos por presuntos fraudes relacionados a la venta ilegal de terrenos.

Uno de los elementos más significativos que observamos durante el trabajo de campo fue que las características del contexto impiden la construcción de redes sociales. Las familias de Barranca Honda tienen pocos espacios aptos para realizar actividades grupales o pasar tiempo libre con sus hijos pequeños. Además, debido a los problemas territoriales, los padres y cuidadores consideran que la gente es desconfiada y en ocasiones puede llegar a ser violenta. Una de las madres entrevistadas comentó al respecto: "Casi, la verdad casi no salgo, no soy de andar en la calle. Siempre estoy aquí en casa con Edson”. (CUPS, 15:51).

La desconfianza y la violencia potencial impiden la conformación de redes 
entre vecinos $y$, en consecuencia, el sentido de pertenencia a la comunidad es muy bajo, en otras palabras, falta capital social. Este concepto, capital social, es definido por Putnam (1993, p. 167) como el conjunto de “aquellas características de una organización social, -tales como la confianza, las normas y las redes-, que son susceptibles de mejorar la eficiencia de una sociedad al facilitar la acción colectiva”.

\section{Embarazo, parto y postparto}

El embarazo es una de las etapas fundamentales en la constitución de la salud y el desarrollo general de los niños. Los cuidados y la atención que recibe la mujer en esta etapa determinan, en buena medida, las condiciones físicas y emocionales con las cuales nacerá el bebé.

La planeación y seguimiento del embarazo es algo que toda mujer, sin excepción, debería realizar. Sin embargo, las mujeres de esta colonia, como en la mayor parte de la población en nuestro país, reconocen que sus embarazos no fueron planeados ni controlados debidamente.

Muchas de ellas reaccionaron nerviosas ante la pregunta sobre la planeación del embarazo, con risas y ademanes que manifestaban inseguridad ante la respuesta ${ }^{1}$.

Por otra parte, también con relación al cuidado durante el embarazo, buena parte de las madres del CAIC contaron con atención médica durante este periodo. Dicha atención fue brindada por clínicas públicas derivadas del Seguro Popular, al que en general las madres caracterizan como un mal servicio.

Al parecer, es sobre todo esta percepción la que las lleva a recurrir a médicos

\footnotetext{
${ }^{1}$ Cabe destacar que la mayoría de los entrevistados fueron padres a edades tempranas, lo que hace evidente su necesidad de información y apoyo a la crianza.
}

privados, curanderas y/o parteras, tal y como lo manifiesta una de las madres entrevistadas del CUPS,

Fui al médico y me dijo que había sufrido un aborto, pero entonces, la verdad, como somos personas que todavía nos curamos a la antigüita, una curandera me dijo que no, que yo estaba embarazada... ¿cómo, si me dijeron que ya lo había perdido? Y dijo: ¡no hija, yo te voy a curar y tu bebé va a nacer, no vayas al médico porque si no, te sacan al bebé que tienes! ... y mire... ¡ahí está mi bebé! (CUPS 5:45).

En muchos casos la atención médica no se dio sino hasta el momento del parto. Sin embargo, la mayoría de las mujeres que presentaron complicaciones, o embarazos de alto riesgo, recibieron atención médica durante la gestación.

Cuando yo empecé con mis sangrados no me llevaron porque dieron de baja a mi esposo por un mes, entonces nosotros pensamos que ya no teníamos el servicio. Fuimos... no sé a dónde me llevó [...] Nos decían que tenía un hematoma, pero pues nunca me dieron los resultados. Solamente me estuvieron atendiendo, porque estuve en el hospital universitario, como ve que mi esposo trabajaba ahí, teníamos seguro por parte del universitario. Y fui y ahí me estuvieron checando, y yo recuerdo que la última vez me dieron una pastilla roja y me dijeron "con esto se tiene que pegar la placenta” porque supuestamente estaba despegada... me la tomo, y con eso (se solucionó el problema). (CAIC 2:78).

Con relación a los cuidados durante el embarazo (ya sea de riesgo o no), éste no resulta ser un tema relevante para ninguno de los dos grupos. Al preguntarles al respecto, les costaba trabajo recordar las fechas y cosas que hacían para procurarse o procurar a su bebé. En general, podemos observar que los cuidados 
prenatales no son óptimos, y esto parece ser derivado de factores tanto económicos como culturales. A este respecto se pone en evidencia la necesidad de reforzar la información sobre la importancia de la planeación y el cuidado durante el embarazo, ya que ello puede ayudar a reducir considerablemente embarazos en adolescentes, riesgos de desnutrición, malformación, complicaciones diversas e incluso prevenir la muerte materna y/o infantil.

\section{Bienestar físico del niño}

Durante el crecimiento, los niños necesitan de ciertas condiciones mínimas para desenvolverse adecuadamente. Si bien la satisfacción de sus necesidades básicas posibilita su desarrollo, cumplir con ello no es suficiente para garantizar un desarrollo óptimo. Es tarea de los padres o cuidadores mantener a los niños sanos y salvos, fuera de situaciones peligrosas que pudieran dañar su integridad y seguridad.

El cuidado de la salud y una vivienda digna son parte primordial de los derechos de los niños debido a que son esenciales para el desarrollo óptimo de sus capacidades físicas y psíquicas. Aspectos considerados en el análisis:

La salud de los niños incluye acciones de promoción, prevención y atención, y son fundamentales en las etapas tempranas de la vida. Si bien las familias de esta colonia cuentan con el seguro popular, en ocasiones no tienen los recursos económicos para adquirir los medicamentos que los niños necesitan:

Pues nació con bajo peso y pues sí lloraba, fue, bueno como te podría decir, por como a los tres [sic], cuatro meses estaba muy baja de peso y tuve que comprar leche de bote, pero pues, ahora sí que, por mis recursos, pues ya no, nada más le di como como tres, tres veces y hasta ahí nada más. (CUPS 3:18).
Aunado a dicha situación, algunos niños que han sufrido accidentes no han sido atendidos de manera apropiada, lo cual incrementa el riesgo de convertirse en un problema mayor de salud para ese niño. Tal es el caso que describe una mamá del CUPS,

[...] la dejé que se viniera con él y dice mi hija que se columpió, estaban columpiándose, entonces ella pasó y le pegaron con el columpio y se convulsionó mi hija, entonces desde ahí le duele mucho luego la cabeza o con cualquier golpe que se dé le duele la cabeza. (CUPS 3:66).

Las condiciones de las viviendas en Barranca Honda suelen ser insalubres e inseguras. Encontramos casas con piso de tierra, con cables expuestos y pelados, segundos pisos y escaleras sin barandales, hacinamiento habitacional, convivencia con animales no domésticos, materiales de construcción dispersos dentro y fuera del hogar, entre otras cosas. Estas características de las viviendas, aunadas al descuido de los padres o cuidadores, han provocado serios accidentes en los niños, entre los cuales destacan: caídas e intoxicaciones.

Derivado de las diferencias en el perfil socioeconómico de las familias (CAIC/ CUPS), en el caso de las primeras, cuentan con casas de concreto y con servicios mínimos como son agua, luz, gas, techo y piso firme. En cambio, para las familias del CUPS el escenario es distinto: en su mayoría habitan casas en condiciones precarias y no cuentan con todos los servicios. La colonia suele tener problemas con los servicios de limpia y recolección de basura, motivo por el cual es común que en las casas y en las calles se acumulen los residuos durante largos períodos de tiempo.

Entre las vías más efectivas para prevenir las enfermedades están los hábitos 
de higiene, la alimentación y las vacunas. En los dos casos estudiados, los padres y cuidadores de Barranca Honda no suelen tener buenas prácticas al respecto. Son frecuentes las enfermedades respiratorias, cutáneas y gastrointestinales debidas a la falta de higiene. Además, en la colonia escasea el agua y no es potable. En el caso de los niños del CUPS, pocos cuentan con baño con regadera, por lo que el baño ocurre una o dos veces a la semana. Si bien se evidencia que los niños del CAIC poseen mejores condiciones, ésta no es una característica que se pueda generalizar ni asimilar al nivel socioeconómico de las familias, así, por ejemplo:

Hay unos [papás] que haga de cuenta, llevan a los niños y los llevan sin desayunar, los llevan, y no por decir "que seas pobre, seas cochino", o sea, no por eso luego los niños van con su uniforme todo arrugado, sucio. A lo menos acá, por lo que tengo a mi suegra, siempre le gusta planchar la ropa, yo llegue acá y todo planchado y todo acomodado. Como le digo a mi hija, "tú tienes que limpiar tus zapatitos para la escuela, porque a ti no te juzgan, a quien juzgan es a tu mamá, porque yo soy, yo soy la señora que no trabaja... i ¿̇y que lleve sus hijos así?!” (CAIC 12:31).

Con respecto a la alimentación, sin embargo, es notoria la diferencia entre ambos grupos. Los niños de CAIC suelen tener disponibles más alimentos, aunque no necesariamente estar mejor alimentados. Muchos de ellos tienen una dieta basada en sopas de pasta, algunas verduras, poca carne y poca fruta. Los padres frecuentemente dan a sus hijos alimentos chatarra, como premio ante una buena conducta.

El caso de los niños del CUPS es más grave, estos pequeños suelen comer muy poco y sin variedad, la mayoría de ellos consume una sola ración de alimento al día, el cual consiste, principalmente, en tortillas de maíz y café. Algunos beben refrescos de cola durante el día, pues dado su bajo costo y alto contenido calórico resulta efectivo para saciar el hambre de los niños de estas familias. En este grupo fueron evidentes los datos de desnutrición: bajo peso y talla, piel con manchas, ojos amarillentos, falta de energía y baja tolerancia al frío, entre otros.

\section{Bienestar psicosocial}

El aspecto psicosocial se refiere a las herramientas psíquicas y sociales que requiere el niño para desenvolverse adecuadamente en el mundo. Los padres y cuidadores son los responsables de brindarle, en sus primeros años de vida, un ambiente de seguridad emocional que le permita crecer seguro y con una autoestima saludable. Aunado a la seguridad emocional, se requiere de relaciones afectivas positivas entre los miembros de la familia, para que el niño no manifieste signos de stress tóxico durante su desarrollo.

En algunas familias se identificaron acontecimientos muy significativos que han impactado directamente en el niño, afectando su desenvolvimiento físico, social y afectivo:

Le afectó mucho la muerte de su tío ... lo asesinaron en la secundaria ... Siempre estaba con él y a todos lados iba con él y se peleaba con él y se venía bien enojado y decía "es que me regañó mi tío", y ya se acostaba; pasaba un ratito y otra vez se iba a buscarlo y jugaba mucho con él, entonces, al ya no verlo, al ya no saber dónde estaba, empezó con que no quería comer, y no quería comer. Nada más estaba sentado, veía tele y nos decía que a dónde estaba su tío, y a qué horas iba a venir, y por qué no venía. (CAIC 15:44).

Las malas experiencias escolares relacionadas al descuido y maltrato por parte 
de las maestras o el personal han implicado que algunas familias desconfíen de los centros educativos, negándose a llevar a sus hijos a la escuela.

Luego dice que por eso ya no quería ir a la guardería, porque dice que allá le daban sus pellizcotes, y dice que le daba la maestra, la otra..., una gordita, dice que luego le daba de patadas atrás, en su colita, y que todavía le decía: -no más donde al rato venga a tu mamá y le digas [lo que te hice]... ¡vas a ver cómo te va a ir mañana! - así le decía. (CAIC 1:93).

Por su parte, el comportamiento de los padres frente a sus hijos, quizá como producto de su propia inmadurez, ha generado que se establezcan relaciones nocivas y conflictivas entre ellos y con los otros niños. La violencia, en mayor o menor grado y en sus diversas formas, parece ser una constante inadvertida como tal por las familias: "Ayer igual me dijo eso la maestra, que a lo mejor ve que nos pegamos y nos jaloneamos y nos gritamos [los padres], y que por eso el niño ve esas cosas, y que por eso él se porta así.” (CAIC 1:25).

En Barranca Honda los niños generan vínculos afectivos más importantes con la madre dado que los padres generalmente se encuentran ausentes, ya sea por trabajo o por falta de apego hacia los menores. Existen casos en los que los padres, madres o cuidadores deben trabajar y no cuentan con familiares cercanos o alguna red de apoyo para el cuidado de los niños durante su ausencia, recurriendo a los internados: "Yo tuve que llevar a mi hija, me la tuve que llevar a un internado para que yo pudiera buscar un trabajo para poderla sacar adelante porque yo no tenía a quién recurrir para tenerla.” (CAIC 1:33).

En cuanto a los castigos, cuando los padres o cuidadores deben aplicar medidas correctivas no suelen recurrir al maltrato o a los golpes, sino que establecen castigos, usualmente, dirigidos a retirar algún beneficio,

[...] le digo: no debes de pegarle a tus compañeritos, debes de obedecer a tus maestras, debes de portarte bien. Le digo: mira mijo, ...mi hija iba a comprar algo y le digo: vete a comprar un Presto barba y te compras un juguito, de esos de a peso, [...] y me dice que no, que le pegue como su papá, y le digo: no te voy a castigar de esa manera, no te quiero pegar, no te quiero golpear porque eres mi hijo y siento feo, estás chiquito y siento feo, le digo: pero hoy no te voy a comprar nada. (CAIC 1:57).

\section{Desarrollo físico}

El desarrollo físico, no sólo abarca las características físicas del crecimiento del niño, del mismo modo hace referencia a la importancia de su alimentación e higiene, $\mathrm{y}$ a la promoción por parte de los cuidadores o los padres de lugares seguros para el juego y la exploración.

Cuando se refieren al desarrollo motor y al lenguaje de los niños, las madres de familia hablan de acciones que parecieran ser espontáneas, es decir, no se observa que existan procesos de estimulación por su parte,

[...] A caminar, este..., pues, empezó en andadera, desde como a los 6 meses empezaba solito a andar, como estaba toda esta zona, pues corría, nada más estaba en los sillones y él iba y venía, o en la parte de allá adentro, que es la cocina... Pero de ahí, este... Lo sacamos un día de la andadera y lo dejamos parado entre los sillones y solito cruzaba de un lugar a otro y ya... (CAIC 15:43).

La mayoría de las familias carecen de hábitos y rutinas establecidos; en el caso de la alimentación, como se indicaba arriba, 
los niños pueden llegar a comer en varias ocasiones 0 , por el contrario, dejar pasar largos periodos de tiempo entre comidas, dependiendo no sólo de la disponibilidad del alimento, sino de los horarios de los hermanos mayores, actividades de la madre, o simplemente, "hasta que les de hambre". El caso del sueño es similar, los horarios son irregulares y dependen del descanso de los padres.

En relación al juego, pocas familias establecen dentro de la rutina diaria tiempo para jugar con sus hijos; los niños generalmente juegan solos o con sus hermanos mayores o personas significativas. "Le gusta jugar con mi suegra [...] juegan con sus carros, a la plastilina, a que según lucha con su abuelito [...]” (CAIC 5:95).

La falta de espacios públicos y recreativos impide la socialización con otros niños. En el caso de los niños del CAIC, tienen la oportunidad de convivir con otros niños y otros adultos en este espacio, sin embargo, la escuela tampoco ha conseguido fomentar la participación y convivencia de la comunidad educativa. Los niños del CUPS suelen convivir únicamente con sus familiares. Los que tienen parientes que viven en la misma Colonia pasan buena parte del tiempo con otros adultos distintos a sus padres. Los que solo tienen a la familia nuclear, pasan los primeros años de vida en la propia casa. Los hermanos mayores de cinco años, que no están al cuidado de ningún adulto, están parte del día en la calle. Suelen andar en bicicleta o jugar al fútbol en las canchas (terrenos de terracería que están ubicados debajo de torres de alta tensión).

\section{Desarrollo mental}

La formación del niño está influenciada por las interacciones que tiene con su ambiente, las posibilidades para explorar, interactuar con otras personas, objetos y espacios. El juego es una herramienta que puede fortalecer el desarrollo de capacidades ejecutivas y habilidades de pensamiento y resolución de problemas, que más tarde impactarán en su aprendizaje.

En algunas familias del CAIC existen buenas condiciones en las interacciones entre los cuidadores y los niños. Las interacciones promueven hábitos saludables, y muestran afecto, cariño, apego y responsabilidad por parte del cuidador:

Cuando me ve ahí que estoy acostada pues se acuesta, se acuesta conmigo porque él es de los que: -mami no me quiero acostar hasta que tú te acuestes, y me dice: -te espero, y luego ando aquí lavando, todavía a veces hasta las 9 de la noche, $y$ no se va a dormir hasta que yo me voy. (CAIC 1:20).

Se identificaron interacciones familiares asociadas a conflictos menores entre las madres y los niños, derivados de caprichos del niño, o ante situaciones que no son cómodas para él. Sin embargo, también se presentaron casos de familias con violencia física o psicológica, lo cual impacta negativamente en el desarrollo emocional de los niños:

Mire lo que pasa es que mi esposo me pegó una vez y mis hijos vieron eso. Entonces yo creo se pone así, de repente, porque como mi esposo me pegó, a él no se le olvida. Luego le dice "es que tú, tú pegas y por eso yo pego, porque tú pegas”. [...] Ese día quién sabe qué le pasó, se le botó la locura, y pues me pegó y mis hijos vieron. (CAIC 1:23).

La televisión es un elemento importante en las actividades de entretenimiento de todas las familias, y dado que los niños permanecen muchas horas en casa, por la falta de espacios 
recreativos, permanecen muchas horas de la tarde frente al televisor, "[...] él es de los que llegan de la escuela, hace su tarea y ve la tele hasta que se duerme..." (CAIC 1:4). Incluso existen familias en las que, a pesar del bajo ingreso, se realizan esfuerzos económicos para adquirir los aparatos nuevos, como consecuencia del apagón digital.

\section{Análisis y discusión}

A partir de los antecedentes del estudio y del análisis de toda la información obtenida acerca de las prácticas de crianza en Barranca Honda, pudimos conocer los principales factores de riesgo para la primera infancia que allí habita, un grupo ciertamente vulnerable pero caracterizado por una serie prácticas que vulneran aún más su situación. Dichos factores de riesgo son tanto externos como internos, es decir, si bien muchos de ellos tienen que ver con el desventajado contexto en el que viven las familias; otros se derivan de las características propias de dichos núcleos parentales; de las creencias, prejuicios o experiencias previas que poseen o no.

Así, en lo que respecta al embarazo, parto y postparto, la falta de asistencia médica apropiada es una constante que pone en riesgo la vida de madres e hijos. Las causas de dicha falta son diversas: las malas condiciones económicas de las familias, su ignorancia respecto de las implicaciones de no recibir dicha atención de forma oportuna, y hasta sus propios prejuicios y creencias al respecto, los cuales los llevan a optar por opciones más baratas y menos ortodoxas, como la curandera.

En ambos grupos se encontró que la mayoría de los padres tienen bajos niveles de escolaridad e iniciaron su paternidad durante la adolescencia, lo cual podría explicar, en cierta medida, la falta de conocimiento y experiencia que rondan sus prácticas parentales. No obstante, la falta de redes de apoyo, como las que brinda la familia extensa (abuelas, tías, o hermanas mayores), parece ser la principal causa de incidencia de ciertas prácticas de riesgo para los niños.

En lo que respecta a su desarrollo físico y mental, lamentablemente las condiciones no parecen estar dadas para su pleno desarrollo. Los padres de familia no poseen la madurez necesaria como para comprender el modo en que sus acciones se ven reflejadas en las de los niños, y cómo éstas pueden ir en detrimento de su pleno desarrollo. Tampoco parecen vislumbrar con claridad la importancia de jugar con ellos y de establecer ciertas rutinas diarias básicas (como aquellas que tienen que ver con el aseo, el sueño y la alimentación) para fomentar su crecimiento físico, mental, emocional y social. Esto parece tener que ver con que la mayoría de las prácticas de crianza están dedicadas a la satisfacción de las necesidades básicas de los hijos.

En general, estas familias tienen rutinas diarias que no favorecen el desarrollo integral de los niños, y dado que hablamos de padres muy jóvenes, es posible que estas creencias tengan que ver con su propia experiencia reciente; es decir, en la mayoría de los casos los padres no conocen otra forma de ser y actuar, y no saben en dónde radica el problema porque, aparentemente, nada de esto representa un problema para ellos en su vida actual.

No encontramos diferencias significativas en las prácticas de crianza entre los grupos, pero sí en las condiciones socioeconómicas, específicamente, las relacionadas con el ingreso económico de las familias. Los padres del grupo A tienen ingresos bajos e irregulares (son albañiles, cargadores, etc.), mientras que los del grupo $\mathrm{B}$ tienen bajos ingresos pero con mayor regularidad (son obreros, comerciantes 
informales, etc.). Estas diferencias se reflejan en las condiciones de las viviendas: en el grupo A, las viviendas tienen piso de tierra, techos de láminas metálicas y algunas no tienen ni baño ni ducha. En el grupo B, casi todas las casas tienen pisos y techos de cemento y tienen baño con ducha. También hay diferencias en la alimentación: en el grupo A, la mayoría de los niños tiene una dieta irregular y precaria en cantidad y calidad, mientras que en el grupo B los niños tienen una dieta con mayor cantidad de alimento, aunque no siempre lo que comen es de buena calidad.

Como hemos dicho, no observamos diferencias significativas en las prácticas de crianza entre ambos grupos. Las características similares en los grupos son que la mayoría de los padres tienen bajos niveles de escolaridad e iniciaron su paternidad durante la adolescencia. En general, estas familias tienen rutinas que no favorecen acciones que promuevan el desarrollo integral de los niños. La mayoría de las prácticas de crianza están dedicadas a la satisfacción de las necesidades básicas de los hijos, y los padres tienen como principal criterio de bienestar el desarrollo físico.

Uno de los elementos más significativos que observamos durante el trabajo de campo fueron las características del contexto que impiden la construcción de redes sociales. Las familias de Barranca Honda tienen pocos espacios aptos para realizar actividades grupales o pasar tiempo libre con sus hijos pequeños. Además, debido a los problemas territoriales, los padres y cuidadores consideran que la gente es desconfiada $\mathrm{y}$ en ocasiones puede llegar a ser violenta. La desconfianza y la violencia potencial impiden la conformación de redes entre vecinos y, en consecuencia, el sentido de pertenencia a la comunidad es muy bajo, en otras palabras, falta capital social.

\section{Reflexiones finales}

Uno de los desafíos afrontados por quienes desarrollan políticas y programas dirigidos a la primera infancia y sus familias, es lograr los mejores resultados intentado conciliar la evidencia científica sobre lo que un niño necesita, con las prácticas y creencias tradicionales efectivas de crianza. (EVANS; MYERS, 1994). Este reto se debe intentar resolver, en la medida de lo posible, antes de que las políticas y los programas se diseñen.

Conocer las condiciones de riesgo en las que viven y crecen los niños y las niñas de Barranca Honda, nos permite comprender mejor aquello que incide en su desarrollo, cuya tendencia muy probablemente generará situaciones de exclusión educativa que a su vez reproducen la desigualdad social. En nuestra opinión, y de muchos expertos, las condiciones de pobreza no son necesariamente condiciones de riesgo para el desarrollo integral de la infancia cuando las familias cuentan con los apoyos necesarios para mejorar sus prácticas de crianza. (FUJIMOTO, 2009; VILLASEÑOR, 2012). En este sentido, las experiencias socioeducativas dirigidas a favorecer las prácticas de crianza de padres y cuidadores han demostrado ser una de las vías más efectivas para promover el desarrollo infantil y disminuir las desigualdades sociales.

En el caso concreto de Barranca Honda, consideramos urgente implementar programas socioeducativos cuyo propósito sea favorecer el desarrollo integral de niños y niñas durante los primeros años de vida, impactando a través del juego en las prácticas de crianza de los cuidadores y en la creación de redes de confianza y apoyo (capital social) de manera tal que se favorezcan el desarrollo de la primera infancia y de la comunidad misma. 


\section{Referências}

BECK, U. La sociedad del riesgo. Hacia una nueva modernidad. Barcelona: Paidós, 1998.

CONEVAL. Diagnóstico del avance en monitoreo y evaluación en las entidades federativas 2013. Ciudad de México: CONEVAL, 2013.

EVANS, J. L.; MYERS, R. G. Childrearing practices: creating programs where traditions and modern practices meet. Coordinators notebook, Leiden, n. 15, p.1-21, aug.1994.

FUJIMOTO, G. El futuro de la educación iberoamericana: ¿es la no escolarización una alternativa? La primera infancia (0-6 años) y su futuro. Madrid: OEI y Fundación Santillana, 2009.

HANINGTON, B; MARTIN, B. Universal methods of design: 100 Ways to research complex problems, develop innovative ideas, and design effective solutions. USA: Rockport Publishers, 2012.

HECKMAN, J. J. Skill formation and the economics of investing in disadvantaged children. Science, v. 312, p. 1900-1902, jun., 2006.

INEGI. (ENADID). Encuesta nacional de la dinámica demográfica. Metodología y tabulados gráficos. Aguascalientes: INEGI, 2010 .

NATIONAL SCIENTIFIC COUNCIL ON THE DEVELOPING CHILD. The science of early childhood development. Disponível em: <http://www.developingchild.net>. Acesso em: 21 jul. 2010.

PUTNAM, R. D. Bowling alone: the collapse and revival of American community. New York: Simon and Schuster, 2001.
SILVA, C.; BURGOS, C. Tiempo mínimo

- conocimiento suficiente: la cuasietnografía sociotécnica en Psicología Social. Psicoperspectivas, Valparaíso, Chile,v.10, n.2, p. 87-108, 2011.

SIRAJ-BLATCHFORD, I.; WOODHEAD, $M$. Effective early childhood programmed. Early childhood in focus. Milton Keynes: The Open University, 2009.

UNESCO. Informe de seguimiento de la EPT en el mundo: bases sólidas: atención y educación de la primera infancia. París: UNESCO, 2007.

UNICEF. Informe anual UNICEF México 2010. México: UNICEF, 2010.

VILLASEÑOR, K. Un buen inicio: indicadores de buenas prácticas de AEPI en Puebla, México. Barcelona, 2012. 209 f. Tesis Doctoral, Departamento de Pedagogía Sistemática y Social. Universidad Autónoma de Barcelona, Barcelona, 2012.

WOODHEAD, M. Changing perspectives on early childhood: theory, research and policy. Background paper prepared for the Education for All Global Monitoring Report 2007. París: UNESCO, 2006. 